by filtration, washed with $3 \%$ hydrochloric acid, dried and ignited. The resulting oxides weighed $0.4286 \mathrm{~g}$. $50 \mathrm{cc}$. of the solution treated in a similar manner gave $0.2 \mathrm{l} 4 \mathrm{I} \mathrm{g}$. These quantities represented 86.36 and $86.40 \%$ respectively, of zirconium and titanium oxides.

Average by selenious acid.............. $86.38 \%$

By cupferron was.................. $86.36 \%$

$0.259 \mathrm{I} \mathrm{g}$. of another sample of zirconia ore gave $0.1939 \mathrm{~g}$. of oxides of zirconium and titanium, or $74.84 \%$, while the average given by cupferron was $74.70 \%$

The method described above was found to be very rapid, simple and accurate for the determination of zirconium. The reagent, selenious acid, is cheap.

The determination of thorium by means of selenious acid and the determination of tantalum and columbium by means of cupferron are being investigated at the present time.

DURHAM, N. H.

[CONTRIBUtTon from the CheMICAL LABORATORY OF THE UNIVERSTTY OF ILIINOIS.]

\title{
TRANSFERENCE NUMBERS OF SODIUM AND POTASSIUM IN MIXED CHLORIDE SOLUTIONS. (PRELIMINARY PAPER.)
}

\author{
By S. A. BRaley and J. L,OWE Halir. \\ Received January 2, 1920.
}

In a study of the equilibria existing between mixed salt solutions and liquid amalgams, Smith and Ball ${ }^{2}$ have noted that, with equivalent mixtures of sodium and potassium chlorides in solution, the ion-fraction of the potassium gradually decreases with increasing total salt concentration. In other studies on sodium-strontium ${ }^{3}$ and potassium-strontium ${ }^{4}$ mixtures similar results have been obtained. Several explanations may be offered to account for this behavior and the present work has been carried out for the purpose of throwing some light upon the subject by comparing the calculated values (on the basis of the isohydric principle) with the determined values of the transference numbers at various equivalent salt concentrations.

G. M. J. McKay, by the use of transference experiments in mixed solutions of potassium chloride and potassium sulfate, $0.2009 \mathrm{~N}$ with respect to each, has shown that the ratio of the ionization factors of the 2 salts in the mixture is $\mathrm{I}, 372$, whereas the ratio as calculated from the

1 The authors wish to thank Dr. T. R. Ball for originally suggesting the problem and starting the work upon it.

${ }^{2}$ Strith and Ball, This Journal, 39, 779 ( 1917 ).

"Smith and Braley, ibid., 39, 1545 (1917).

${ }^{4}$ Smith and Rees, ibid., 40, 1802 (1918).

sMeKay, ibid., 33, 308 (r9o8). 
conductance of the solution on the basis of the isohydric principle is I. 275 . Also, in general, calculations based on conductivity do not yield values in agreement with those obtained by other methods.

Many examples of the double salts in which the halides appear are known, such as $\mathrm{K}_{2} \mathrm{PtCl}_{6}, \mathrm{KCl} \cdot \mathrm{MgCl}_{2} 6 \mathrm{H}_{2} \mathrm{O}, \mathrm{Na}_{3} \mathrm{AlFe}, 2 \mathrm{NaCl} \mathrm{Al}_{2} \mathrm{Cl}_{6}$, the potassium iodide complexes with the iodides of cadmium, mercury, zinc, bismuth, etc. It is, therefore, not altogether rash to expect that the alkali halides form complexes with each other in solution, especially as their ions are known to be hydrated. ${ }^{1}$

On this assumption we would then have one of the metals showing a decrease of transference due to the back transference of the metal in the negative complex.

\section{Theoretical.}

The transference number of an ion in solution is

$$
T \oplus=K C \oplus+U \oplus=K^{\prime} C \oplus+\Lambda \oplus
$$

where $\oplus$ represents the ion.

But

Also

$$
C \oplus=C_{\text {salt }} \alpha_{\text {salt }}
$$

$$
\alpha=\Lambda / \Lambda_{0} .
$$

Therefore, the ratio of the transference numbers of the sodium and potassium ions in the solution will be

$$
\frac{T_{\mathrm{Na}}}{T_{\mathrm{K}}}=K \frac{\Lambda \mathrm{NaCl} / \Lambda_{0} \mathrm{NaCl} \cdot \Lambda_{\mathrm{Na}} \oplus}{\Lambda \mathrm{KCl} / \Lambda_{0} \mathrm{KCl} \cdot \Lambda_{\mathrm{K}} \oplus}
$$

Since equivalent ion conductances are known only at infinite dilution, it is necessary to assume, as McKay ${ }^{2}$ does, that the ratio of these values for sodium and potassium ions is the same as at infinite dilution. Also, as Washburn ${ }^{2}$ and McKay $^{2}$ both observe that in the case of 2 strong electrolytes in a mixed solution, ionization calculations based on total salt concentration agree quite closely with those based on the common ion concentration, the former will be used in the calculations. As viscosity corrections would be applied alike to numerator and denominator, they will be omitted.

The value of the proportionality constant $K$ in Equation 4 may be readily calculated, for at infinite dilution the ratio $\frac{\Lambda \mathrm{NaCl} / \Lambda_{\circ} \mathrm{NaCl}}{\Lambda \mathrm{KCl} / \Lambda_{\circ} \mathrm{KCl}}$ becomes equal to one, hence $\frac{T_{\mathrm{Na}}}{T_{\mathrm{K}}}=K \frac{\Lambda_{\mathrm{Na}} \oplus}{\Lambda_{\mathrm{K}} \oplus}$, in which case $K$ becomes equal to
I. 17 .

${ }^{1}$ E. W. Washburn, Tech. Quart., 21, 288 (1908).

${ }^{2}$ Loc. cit. 
Materials, Apparatus and Method of Experimentation.

r. Materials.-(a) Sodium and Potassium Chlorides.-These salts were prepared by twice precipitating good commercial grades of the saits in aqueous solution with hydrogen chloride, centrifuging and drying in an electric muffle at about $75^{\circ}$. They were stored in glass-stoppered bottles, and again dried in the muffle in quantities as used.

(b) Conlometer Materials.-I5 g. of crystalline C. P. silver nitrate was dissolved in I5o cc. of distilled water shortly before using. This amount of solution just conveniently filled both coulometers.

A pure grade of silver ( $99.9 \%$ fine) was used for anodes. The silver was melted on a bone-ash cupel by means of a blast lamp. A piece of heavy silver wire was inserted into the molten silver button, and the mass allowed to solidify.

A weighed platinum dish was used as a cathode.

(c) Silver Chloride for Cathode.-The silver nitrate solution from the coulometers used in the previous run was kept in a dark place to be used in preparation of silver chloride for the succeeding run. It was filtered, heated to boiling, and precipitated with a hot solution of a little more than the theoretical amount of sodium chloride. The adherent pasty precipitate was finely disintegrated with a glass spatula, washed by decantation 2 or 3 times, and finally transferred to a filter and thoroughly washed. The precipitate was then washed with some of the stock solution of mixed chlorides and transferred to the apparatus.

(d) The Anode.-The anode was prepared essentially as was the "Type B Anode" described by Washburn ${ }^{1}$ for use in transference experiments.

2. Apparatus and Method of Experimentation.-(a) Apparatus.-The same transference apparatus was used as that used and fully described by $\mathrm{E}$. W. Washburn ${ }^{1}$ for the measurement of transference numbers in simple solution. The special coulometer stands were equipped with a glass rod support.

(b) Method of Manipulation.-Essentially the same procedure was followed as that described by Washburn. ${ }^{1}$

(c) Pipets, Burets, and Weights.-The pipets and burets used were calibrated by the Bureau of Standards and the weights were calibrated against a standard set and proper corrections applied.

(d) Thermostat.-A large Freas thermostat electrically controlled was filled with water to a depth sufficient to cover the horizontal section of the apparatus. The temperature was accurate to within $\mathrm{a} 0 . \mathrm{r}^{\circ}$ and constant to within $0.0 \mathrm{r}^{\circ}$.

3. Analysis and Calculations.-(a) Analysis.-Duplicate samples from the end portions and one from each of the 3 middle portions were transferred to small porcelain evaporating dishes for a sodium-potassium

1 Loc, cit. 
determination. At the same time an entire duplicate set of samples was transferted to resistance glass beakers (600 cc. capacity) for a Volhard volumetric determination of chlorine. This analysis was used merely as a check on the total equivalents of salt as found by the chloroplatinate method.

The sodium-potassium determination was carried out by the chloroplatinate method as described by Ball, ${ }^{1}$ with the exception that methyl alcohol (redistilled on a steam bath) was used instead of ethyl alcohol as a separating medium. Care was taken to allow the last traces of the methyl alcohol to evaporate, however, before washing down the filter with hot water and evaporating on the steam bath, as the alcohol exerts a strong reducing effect on the platinum salts when heated. The chloroplatinic acid was also prepared as described by Ball.

(b) Calculations.-The factor 0.3056 was applied to the weight of potassium chloroplatinate to obtain the potassium chloride. Ordinary transference calculations were applied using atomic weights as follows: $\mathrm{Na}=23.0 ; \mathrm{K}=39 . \mathrm{I} ; \mathrm{Cl}=35.46 ;$ and $\mathrm{Ag}=107.88$.

\section{Experimental Data.}

The following table shows the experimental and theoretical values obtained. The basis of comparison of the obtained and theoretical values is the ratio of $T_{\mathrm{Na}} / T_{\mathrm{K}}$, as shown in Equation 4. Conductance data of reliable character covering the range of concentration used, at $25^{\circ}$, were difficult to find. However, values for $\Lambda$ determined by Kohlrausch at $18^{\circ}$ were corrected to $25^{\circ}$ and the values desired were obtained by careful graphical interpolation.

No values are given for the cathode portions as these results did not agree in the least with themselves or with the anode portions. No explanation can be offered at this time but the difference was probably due to manipulation in removing the portion from the silver chloride electrode or to some side reactions on the part of the electrode itself. This matter will be investigated and in this preliminary paper only the anode values will be considered.

TABI, I.

Temp. $25^{\circ}$.

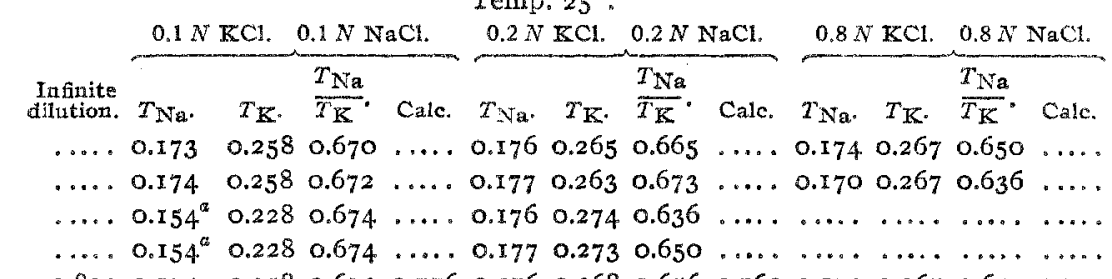

$\begin{array}{lllllllllllllllllll}A v_{0}, & 0.802 & 0.174 & 0.258 & 0.673 & 0.776 & 0.176 & 0.268 & 0.656 & 0.760 & 0.172 & 0.267 & 0.643 & 0.700\end{array}$

a These two values at total concentration of $0.2 \mathrm{~N}$ are evidently low, but this is tundoubtedly due to experimental error as the ratios are the same.

1 Loc. cit. 
It should be noted that a small difference in the transference numbers of the ions themselves makes a large error in the ratio, and because of this fact the checks on the observed ratios are very close and the difference between the observed and calculated values the more siguificant.

In Fig. I the calculated ratios and the observed ratios have been plotted against the total concentration. It is readily seen that while the theoretical ratio is always higher than the experimental the difference decreases as a function of the total concentration. Also, the experimental curve has been extrapolated to the theoretical value at infinite dilution for at this point all complicating factors would be removed and the theoretical value for a simple solution would be obtained.

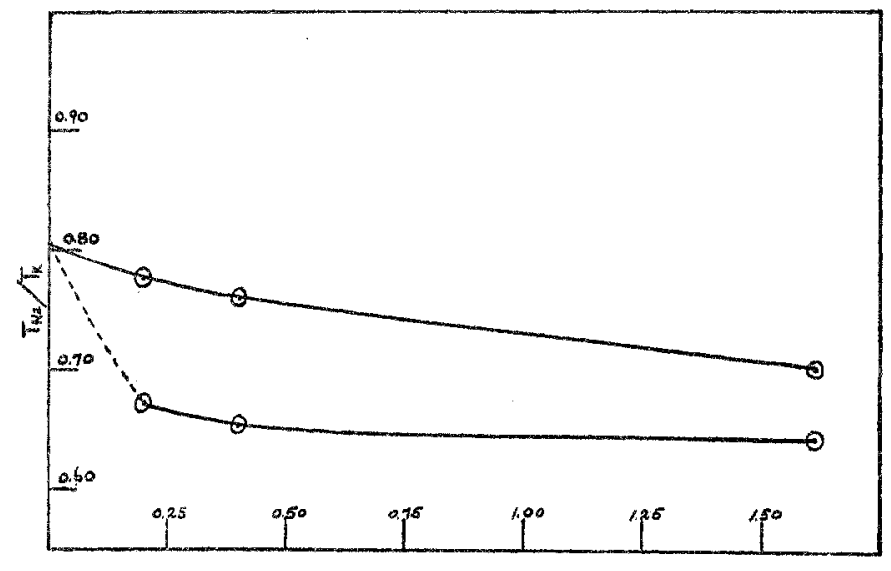

Total Concentration in mols/liter.

Fig. I,-Curves showing variation of $T_{\mathrm{Na}} / T_{\mathrm{K}}$ with total concentration. Upper curve showing calculated values. Lower curve showing determined values.

These results indicate that, on the whole, more potassium was transferred than the theory would indicate, but that over the range studied, that is, form a total concentration of $0.2 \mathrm{~N}$ to $1.6 \mathrm{~N}$ that more sodium is being transferred proportionally than potassium, since over this tange the 2 curves tend to converge with increasing concentration. In other words, from a total concentration of $0.2 N$ to $1.6 \mathrm{~N}$ the sodium gains "transference advantage" with increased concentration. Such an "advantage" may be due to the following possible causes-( $I$ ) a direct increase of the sodium ion fraction, (2) a relative increase of the sodium ion mobility, (3) the entrance of sodintn in superior amounts into positive aggregates, and (4) a "back transference" of potassium in the form of a complex negative ion.

It is interesting to note that the first possibility is in accordance with the results obtained by Ball and Smith, and that the fourth possibility 
would indirectly lead to the same condition, for if potassium goes into complex negative ion formation, the simple sodium ion fraction is correspondingly increased. In connection with the second possibility, Washburn has found that the sodium ion is $50 \%$ more highly hydrated than the potassium ion. And because of the resulting more voluminous and unwieldly character of the sodium ion, which is further evidenced by its much lower ion conductance and transference number, it is reasonable to believe that its ion mobility would be slowed up more, with increasing viscosity, than the potassium ion mobility. This fact undoubtedly accounts for the very sharp break in the determined ratio when extrapolating from a total concentration of $0.2 \mathrm{~N}$ to infinite dilution, as it would be over this range that the hydration would take place to the greatest extent and so exceed the change due to the other factors. If this were the only factor entering, however, it should continue and the curves in Fig. I would continue to diverge; therefore, we must conclude that other factors have entered which overcome this one.

If we assume the formation of complexes in order to account for the results obtained, we of course have none other than a qualitative indication of their nature. In order to satisfy the results obtained in Stmith and Ball's ${ }^{1}$ work and in this investigation, the complex must be of such character as to involve an excessive transference of sodium and a diminution of the simple potassium ion fraction, both of which become more pronounced with increase of concentration above $0.2 \mathrm{~N}$. Mention was made in the third possible cause given above of the possibility of sodium entering in superior amounts in positive aggregates. This condition might be satisfied by the possible interpolation of sodium chloride in place of the water of hydration of the potassium ion. But as a greater opportunity is afforded for the interpolation of potassium in the more highly hydrated sodium ion, and as the removal of free sodium chloride would cut down the sodium ion fraction rather than the potassium, this possibility is inconsistent.

The simplest complex formation possibie is $\mathrm{NaCl} \cdot \mathrm{KCl}$, which may be expressed either as $\mathrm{K}\left(\mathrm{NaCl}_{2}\right)$ or $\mathrm{Na}\left(\mathrm{KCl}_{2}\right)$, and which may ionize to either free potassium ion or free sodium ion. If it should be the first and ionize thus,

$$
\mathrm{K}\left(\mathrm{NaCl}_{2}\right) \rightleftharpoons \mathrm{K}^{+}+\mathrm{NaCl}_{2}^{-},
$$

the simple sodium ion fraction would be relatively decreased and a "back transference" of sodium would occur, both of which are contrary to results.

But if it should ionize thus,

$$
\mathrm{Na}\left(\mathrm{KCl}_{2}\right) \rightleftharpoons \mathrm{Na}^{+}+\mathrm{KCl}_{2}-
$$

1 Loc. cit. 
the sodium ion fraction would be relatively increased, and a "back transference" of potassium would occur, both of which conditions would give an additive tendency to explain the results obtained in both investigations, and which satisfy both the first and fourth possible causes mentioned.

\section{Summary.}

It has been shown that over a range of total concentration from $0.2 \mathrm{~N}$ to r. $6 \mathrm{~N}$ with equivalent mixtures of sodium and potassium chlorides a relatively higher amount of sodium is transferred than is called for by calculation, on the basis of the isohydric principle, from the conductance of the simple salt solutions.

The increase of sodium transferred, over this range of concentration, is roughly a linear function of the total salt concentration.

The results are in harmony with those of Smith and Ball, ${ }^{1}$ according to which they find that the sodium ion-fraction increases with increase of total salt concentration over this same range of concentration.

The results may be explained on the basis of complex formation, the simplest form of which, in accordance with these results, may be expressed thus,

URBANA, IL,LNOLS.

$$
\mathrm{NaCl}+\mathrm{KCl} \rightleftharpoons \mathrm{Na}\left(\mathrm{KCl}_{2}\right) \rightleftharpoons \mathrm{Na}^{+}+\mathrm{KCl}_{2}-
$$

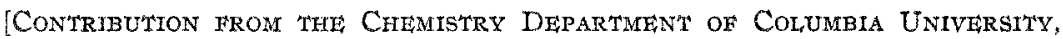
No. 337.1

\section{THE VISCOSITY OF LIQUIDS. IV. IDEAL MIXTURES OF THE TYPES ETHER-ETHER AND ESTER-ESTER. \\ JAMES KENDALL AND ALEXANDER HOLLAND WRIGHT. Received Aptil 27, 1920 .}

Previous attempts to derive a general equation for representing the viscosities of ideal liquid mixtures have been critically examined, and new lines of attack discussed, in earlier papers of this series. In the first article, $^{2}$ all existent formulas were tested by means of the available experimental data and a modification of an equation proposed by Arrhenius ${ }^{3}$ was shown to give most satisfactory agreement. This equation may be written

$$
\log \eta=x \log \eta_{1}+(1-x) \log \eta_{2}
$$

(where $\eta_{1}$ and $\eta_{2}$ are the viscosities of the pure components, and the composition, $x$, is expressed in molecular fractions). Later investigation by Egner ${ }^{4}$ has confirmed the approximate accordance of this formula with the results of experiment.

Loc. cit.

"Kendall, Medd. $K$. Vetenskapsakad. Nobelinsi, 2, No. 25 (1913).

"Arrhenius, Z. physik. Chem., I, 285 (1887).

- Egner, Medd. K. Vetenskapsakad, Nobelinst. 3, No. 22 (1917). 